\title{
Meshing Performance Analysis of Spiral Bevel Gears Based on Assembly Misalignment
}

\author{
Hepeng $\mathrm{Xia}^{1, \mathrm{a}}$, Xiaozhong Deng ${ }^{1, \mathrm{a}}$ and Aijun $\mathrm{Xu}^{3, \mathrm{c}}$ \\ ${ }^{1}$ Collaborative Innovation Center of Manufacture of Advanced Machinery Equipment advanced \\ manufacturing of Henan province ,, Luoyang 471003, China \\ ${ }^{2}$ Collaborative Innovation Center of Manufacture of Advanced Machinery Equipment advanced \\ manufacturing of Henan province ,, Luoyang 471003, China \\ ${ }^{3}$ Electromechanic Engineering College, Henan University of Science and Technology, Luoyang \\ 471003, China \\ (a2295391661@qq.com, bdxz01@163.com, $\left.{ }^{\mathrm{c}} 13938849433 @ 163 . c o m\right)$
}

\begin{abstract}
Keywords: Spiral bevel gears; Assembly misalignment; Tooth contact analysis; Meshing performance
\end{abstract}

\begin{abstract}
The influence of different assembly misalignment on the meshing performance of spiral bevel gears was studied. Based on the principle of local synthesis, the meshing model of spiral bevel gears is established. The tooth contact analysis of spiral bevel gears is introduced. Taking a pair of spiral bevel gears as an example, the influence of assembly misalignment on the meshing performance of gears was studied in the range of error variation. The research results are of great significance for the rapid determination of the optimal installation distance of spiral bevel gears.
\end{abstract}

\section{Introduction}

Spiral bevel gears are widely used in the field of Aeronautics and Astronautics as a kind of key transmission component because of its high coincidence and stable transmission. In the practical application of engineering, the meshing position of spiral bevel gears can not reach the ideal state due to machining error, assembly misalignment and support deformation, and there will always be some transmission error. Transmission error can affect the meshing performance of gear, resulting in poor tooth contact, vibration, reduction of transmission stability, and assembly misalignment is the key factors affecting the transmission error of spiral bevel gears. Prof. Zhonghou Wang ${ }^{[1]}$ put forward a tooth contact analysis (TCA) method based on high precision digital real tooth surface of spiral bevel gears. The feasibility and effectiveness of this method can be verified through the comparison of TCA software analysis results by Gleason corporation and tooth wear test results. Prof. Guanglei $\mathrm{Liu}^{[2]}$ proposed a generally modified-roll (GMR) as an improved method of the traditional local synthesis for the optimization of the transmission error function of spiral bevel gears in comparison with the desired curve. Prof. JinFu $\mathrm{Du}^{[3]}$ proposed the digital gear surfaces that exactly approximate real tooth surfaces are obtained by fitting the measured discrete points with non-uniform rational B-spline (NURBS) curve. A tooth contact analysis (TCA) of digital gear surfaces is carried out and the feasibility of the proposed method is verified through a comparison between digital gear surface TCA and rolling test of a high-speed axle gear pair. Prof. Zongde Fang $^{[4]}$ provided tooth contact analysis algorithm with consideration of alignment errors. Taking a pair of spiral bevel gears as an example, the feasibility of tolerance optimization method was verified by comparing the meshing and transmission errors before and after optimization. Prof. Yaobin Zhuo ${ }^{[5]}$ presented a method for the global optimization of the tooth contact pattern and transmission error of spiral bevel and hypoid gears, and a finite element model of a simplified hypoid gear drive system is established and its quasi-static meshing characteristics analyzed. Suitable contact area meeting design requirements is a common method of evaluating meshing performance, but the evaluation of spiral bevel gears meshing performance also need to refer to transmission error curve. The research on the influence of assembly misalignment on gear meshing performance has seldom mentioned the range of variation of assembly misalignment at home and 
abroad.

On the basis of theoretical design of tooth surface, the tooth contact analysis (TCA) of the designed tooth surface is firstly carried out to obtain the simulation results of the contact area and the transmission error curve of the tooth surface. On this basis, this paper selected axial error of pinion, axial error of gear, offset error of axis and error of axis shaft error as factors, regarded contact boundary point and transmission error curve of amplitude as objectives of design, and studied that the effect of assembly misalignment on meshing performance of spiral bevel gears.

\section{Meshing Model Based on Assembly Misalignment}

Machining models of pinion and gear are established individually, and two wheel tooth surface equations are obtained. The position vectors and the normal vectors of pinion and gear are represented in the meshing coordinate system. The calculation method of meshing model is put forward in document [7] In the process of assembling spiral bevel gears, assembly misalignment including axial error of pinion $\Delta H$, axial error of gear $\Delta J$, offset error of axis $\Delta V$ and axis shaft error $\Delta \Sigma$. In this paper, $S_{1}\left(O_{1} x_{1} y_{1} z_{1}\right)$ represents the coordinate system connected with pinion. $S_{2}$ $\left(O_{2} x_{2} y_{2} z_{2}\right)$ represents the coordinate system connected with gear. $S_{h}\left(O_{h} x_{h} y_{h} z_{h}\right)$ stands for the fixed coordinate system. $S_{d}\left(O_{d} x_{d} y_{d} z_{d}\right)$ represents the coordinate system connected with the $S_{h}$. The coordinate transformation matrices are shown in following equations respectively.

$$
\begin{gathered}
M_{h 1}=\left[\begin{array}{cccc}
1 & 0 & 0 & \Delta H \\
0 & \cos \varphi_{1} & -\sin \varphi_{1} & 0 \\
0 & \sin \varphi_{1} & \cos \varphi_{1} & 0 \\
0 & 0 & 0 & 1
\end{array}\right] \\
M_{h 2}=\left[\begin{array}{cccc}
\cos (\Sigma+\Delta \Sigma) & -\sin \varphi_{2} \cdot \sin (\Sigma+\Delta \Sigma) & -\cos \varphi_{2} \cdot \sin (\Sigma+\Delta \Sigma) & \Delta J \cdot \cos (\Sigma+\Delta \Sigma) \\
0 & -\cos \varphi & \sin \varphi_{2} & \Delta V \\
-\sin (\Sigma+\Delta \Sigma) & -\sin \varphi_{2} \cdot \cos (\Sigma+\Delta \Sigma) & -\cos \varphi_{2} \cdot \cos (\Sigma+\Delta \Sigma) & -\Delta J \cdot \sin (\Sigma+\Delta \Sigma) \\
0 & 0 & 0 & 1
\end{array}\right]
\end{gathered}
$$

In above equations, $\varphi_{1}$ is the angle of rotation of the pinion, $\Sigma$ is the axis shaft angle, and $\varphi_{2}$ is the angle of rotation of gear.
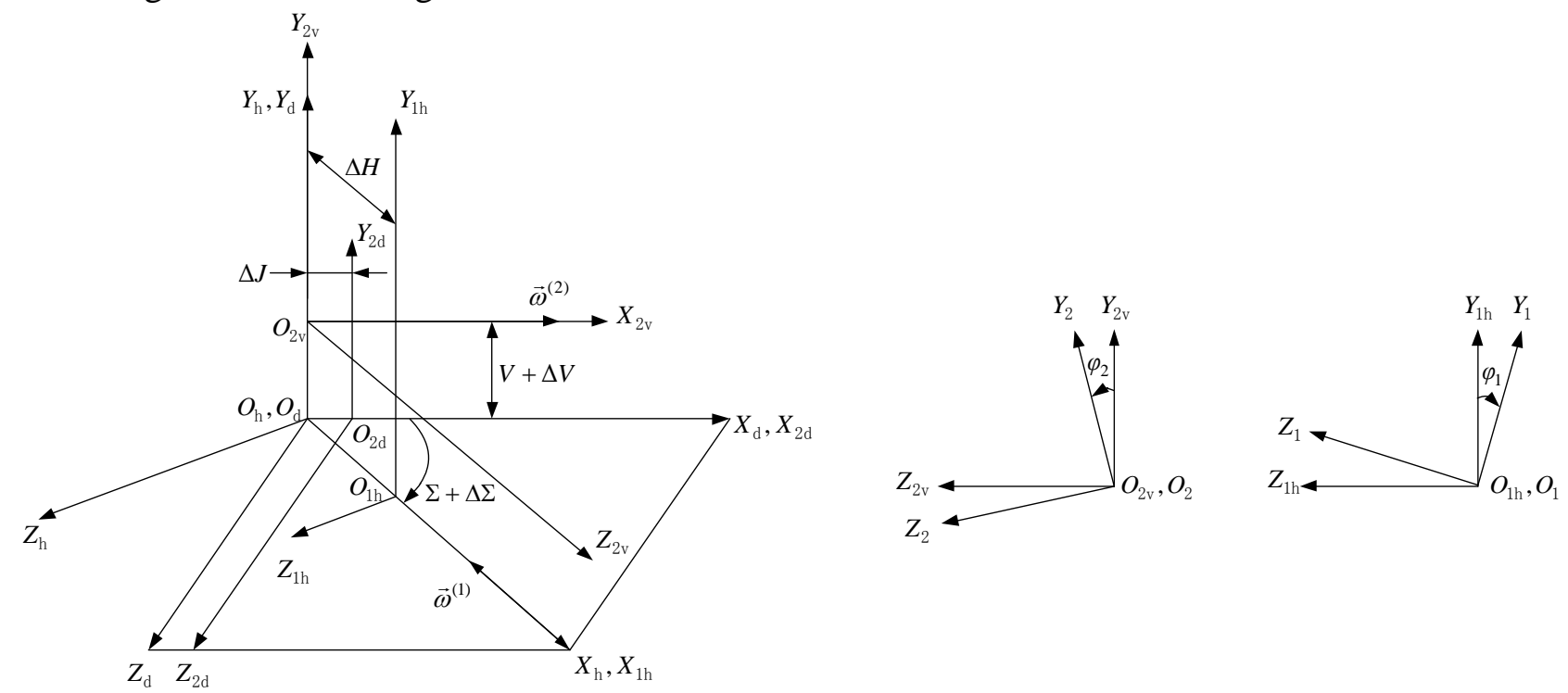

Figure 1. Meshing coordinate system with assembly misalignment 
The tooth surface equations and normal vectors of spiral bevel gears are respectively converted into fixed coordinate system $S_{h}$, and the position vector and unit normal vector of spiral bevel gear in fixed coordinate system are obtained.

$$
\left\{\begin{array}{c}
\vec{r}_{h}^{(1)}\left(\theta_{1}^{*}, \varphi_{p}{ }^{*}, \varphi_{1}, \Delta H\right)=M_{h 1} \vec{r}_{1}\left(\theta_{1}^{*}, \varphi_{p}^{*}\right) \\
\vec{n}_{h}^{(1)}\left(\theta_{1}^{*}, \varphi_{p}^{*}, \varphi_{1}\right)=L_{h 1} \vec{n}_{1}\left(\theta_{1}^{*}, \varphi_{p}^{*}\right) \\
\vec{r}_{h}^{(2)}\left(\theta_{2}^{*}, \varphi_{g}^{*}, \varphi_{2}, \Delta J, \Delta V, \Delta \Sigma\right)=M_{h d} M_{d 2} \vec{r}_{2}\left(\theta_{2}{ }^{*}, \varphi_{g}{ }^{*}\right) \\
\vec{n}_{h}^{(2)}\left(\theta_{2}^{*}, \varphi_{g}{ }^{*}, \varphi_{2}\right)=L_{h d} L_{d 2} \vec{n}_{2}\left(\theta_{2}{ }^{*}, \varphi_{g}{ }^{*}\right)
\end{array}\right.
$$

In the Eq.4, $\theta_{1}^{*}$ is generating the tooth surface of the gear cutter angle. $\theta_{2}^{*}$ is generating tooth surface of cutter angle. $\varphi_{p}{ }^{*}$ is generating the tooth surface of the gear when the cradle angle. $\varphi_{g}{ }^{*}$ is generating the gear tooth surface shake Taiwan angle. $L_{h d}$ is the first $3 \times 3$ order sub matrix of $M_{h d}$ and $L_{d 2}$ is the first $3 \times 3$ order sub matrix of $M_{d 2}$.

According to document [8], by setting the different values of the angle of rotation of the pinion which is expressed as $\varphi_{1}{ }^{\prime}$, the corresponding angle of rotation of the gear $\varphi_{2}{ }^{\prime}$ can be obtained, and the transmission error of the pair of spiral bevel gears is expressed as:

$$
\Delta \varphi=\left(\varphi_{2}{ }^{\prime}-\varphi_{20}\right)-\frac{z_{1}}{z_{2}}\left(\varphi_{1}{ }^{\prime}-\varphi_{10}\right)
$$

In the Eq.7, $\varphi_{1}{ }^{\prime}$ is the current angle of the pinion, $\varphi_{2}{ }^{\prime}$ is the current angle of the gear, $\varphi_{10}$ is the initial angle of rotation of the pinion, $\varphi_{20}$ is the the initial angle of rotation of the gear, $z_{1}$ is the number of teeth of pinion and $z_{2}$ is the number of teeth of gear.

\section{An Example}

Taking a pair of spiral bevel gears as the object of the study, the relationship between the assembly misalignment and the meshing performance of the spiral bevel gears is obtained through tooth contact analysis with assembly misalignment. The geometric parameters of spiral bevel gears are shown in table 1. According to the above process, the TCA simulation results under the assembly misalignment are obtained by taking the concave of the pinion and the the convex of the gear as an example.

Table 1 Geometric parameters of spiral bevel gears

\begin{tabular}{lccc}
\hline Parameter name & pinion & gear \\
\hline $\begin{array}{l}\text { modulus } \\
\text { the number of teeth }\end{array}$ & 25 & 5.5 & 32 \\
$\begin{array}{l}\text { spiral angle }\left[{ }^{\circ}\right] \\
\text { direction of rotation }\end{array}$ & left-hand & 35 & right-hand \\
$\begin{array}{l}\text { axis shaft angle }\left[{ }^{\circ}\right] \\
\text { pitch cone angle }\left[{ }^{\circ}\right]\end{array}$ & 37.9987 & 90 & 52.0013 \\
$\begin{array}{l}\text { Outer cone distance }[\mathrm{mm}] \\
\text { width of tooth }[\mathrm{mm}]\end{array}$ & \multicolumn{3}{c}{111.6717} \\
profile angle $\left[{ }^{\circ}\right]$ & 18 & 45 & 22 \\
\hline
\end{tabular}

According to document [9], the gear contact analysis (TCA) of the above mentioned spiral bevel gears is carried out within the range of the variation of assembly misalignment. When the initial contact point of the surface of the gear is determined, the installation location of the gear pair is determined accordingly. Under the condition that the gear pairs are fixed, the spiral bevel gears obtain a series of contact points from the start point of meshing to the end point of meshing, and the set of these contact points is the contact trace. In this paper, the influence of the assembly misalignment on the contact area is represented by the movement of the midpoint contact traces in the contact area. By the Mat lab program, the position of contact points of the tooth surface under 
the condition of assembly misalignment is extracted, and the coordinates are plotted on the same tooth surface, as shown in figure 2.

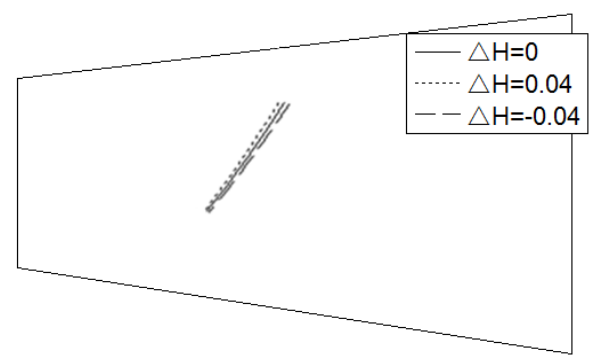

a) The influence of axial error of pinion

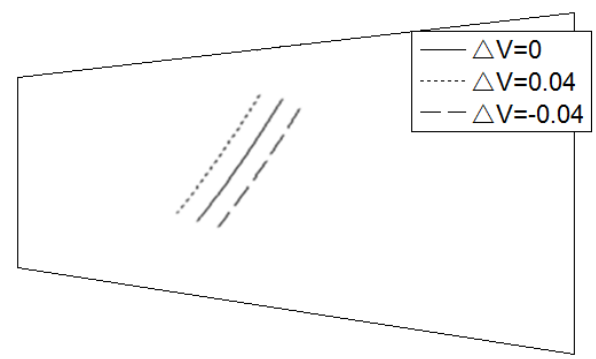

b) The influence of offset error of axis

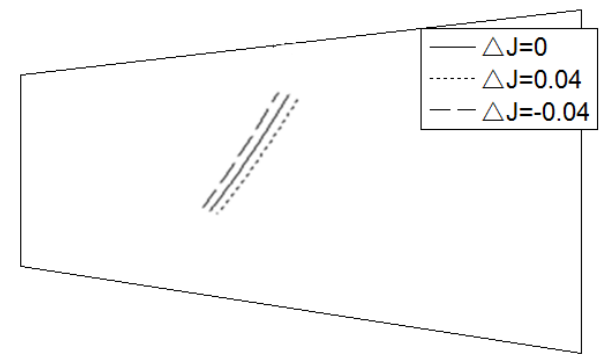

b) The influence of axial error of gear

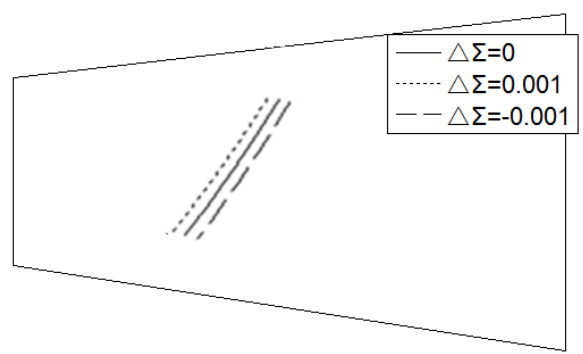

d) The influence of axis shaft error

Figure 2 The influence of assembly misalignment on contact trace of tooth surface

The transmission error curve can reflect the meshing performance of the spiral bevel gears on the other hand. In the analysis of tooth surface contact, the transmission ratio of the reference point of the spiral bevel gears pair is equal to the theoretical transmission ratio of $i$, while the transmission ratio of the other contact points is not necessarily equal to $i$. The transmission error curve is generally used to represent the difference between actual angle of rotation of gear and the theoretical value.

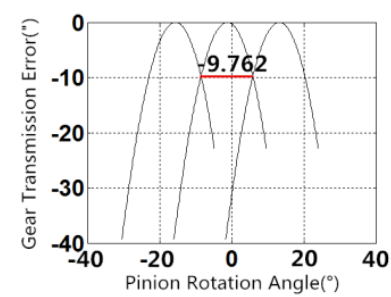

Figure 3 Transmission error curve without assembly misalignment

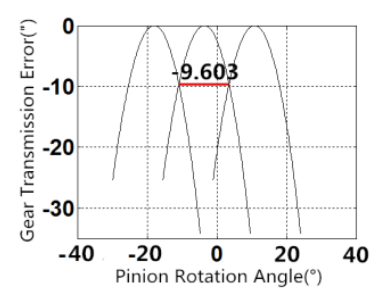

a) $\Delta H=0.04 m m$

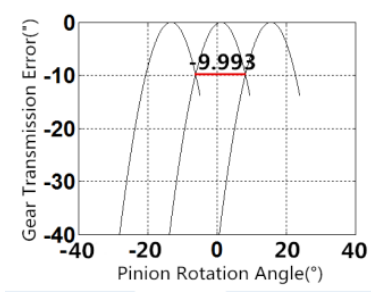

b) $\Delta H=-0.04 \mathrm{~mm}$

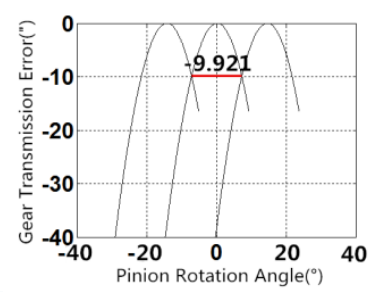

c) $\Delta J=0.04 \mathrm{~mm}$

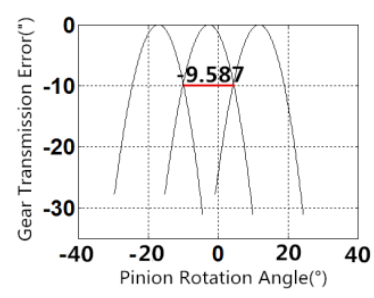

d) $\Delta J=-0.04 \mathrm{~mm}$

Figure 4 Transmission error curve with axial error of pinion or gear 


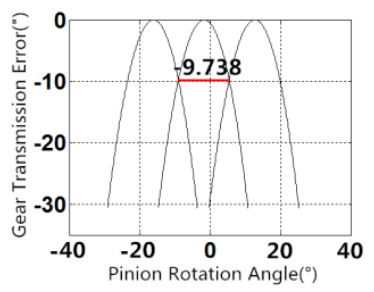

a) $\Delta V=0.04 \mathrm{~mm}$

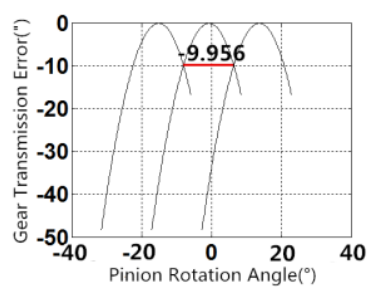

b) $\Delta V=-0.04 m m$

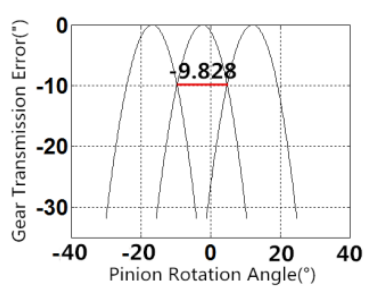

c) $\Delta \Sigma=0.001 \mathrm{rad}$

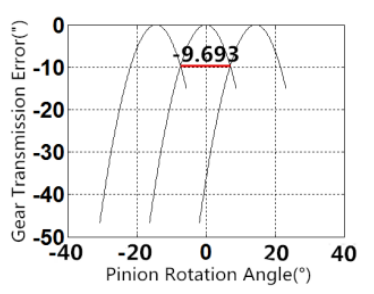

d) $\Delta \Sigma=-0.001 \mathrm{rad}$

Figure 5 The transmission error curve with offset error of axis or axis shaft error

Taking the pair of spiral bevel gears used in this paper as an example. Make a rule that the midpoint of contact trace moving to the toe of tooth is negative, moving to the heel of tooth is positive. The result can be obtained by Figure 2:

Table 2 The relationship of different assembly misalignment on the midpoint of contact traces

\begin{tabular}{lc}
\hline $\begin{array}{l}\text { Adjusting value of } \\
\text { assembly misalignment }\end{array}$ & $\begin{array}{c}\text { Midpoint movement of } \\
\text { contact trace }\end{array}$ \\
\hline$\Delta H=0.04 \mathrm{~mm}$ & $-0.332 \mathrm{~mm}$ \\
$\Delta H=-0.04 \mathrm{~mm}$ & $0.341 \mathrm{~mm}$ \\
$\Delta J=0.04 \mathrm{~mm}$ & $0.771 \mathrm{~mm}$ \\
$\Delta J=-0.04 \mathrm{~mm}$ & $-0.791 \mathrm{~mm}$ \\
$\Delta V=0.04 \mathrm{~mm}$ & $-1.766 \mathrm{~mm}$ \\
$\Delta V=-0.04 \mathrm{~mm}$ & $1.647 \mathrm{~mm}$ \\
$\Delta \Sigma=0.001 \mathrm{rad}$ & $-0.712 \mathrm{~mm}$ \\
$\Delta \Sigma=-0.001 \mathrm{rad}$ & $0.728 \mathrm{~mm}$ \\
\hline
\end{tabular}

The influence of the variation of the four assembly misalignment on the location of contact area is as follows:

axis shaft error > offset error of axis > axial error of gear > axial error of pinion

Taking the pair of spiral bevel gears used in this paper as an example. Make a rule that the amplitude of the transmission error curve increasing is negative, and the amplitude decreasing is negative. The results can be obtained by Figure 3:

Table 3 The influence of different assembly misalignment on transmission error

\begin{tabular}{lc}
\hline $\begin{array}{l}\text { Adjusting value of } \\
\text { assembly misalignment }\end{array}$ & $\begin{array}{c}\text { Amplitude variation of } \\
\text { transmission error curve }\end{array}$ \\
\hline$\Delta H=0.04 \mathrm{~mm}$ & $-0.159 "$ \\
$\Delta H=-0.04 \mathrm{~mm}$ & $0.231 ”$ \\
$\Delta J=0.04 \mathrm{~mm}$ & $0.154 ”$ \\
$\Delta J=-0.04 \mathrm{~mm}$ & $-0.17{ }^{\prime \prime}$ \\
$\Delta V=0.04 \mathrm{~mm}$ & $-0.024 ”$ \\
$\Delta V=-0.04 \mathrm{~mm}$ & $0.192 ”$ \\
$\Delta \Sigma=0.001 \mathrm{rad}$ & $-0.066 ”$ \\
$\Delta \Sigma=-0.001 \mathrm{rad}$ & $0.069 ”$ \\
\hline
\end{tabular}

The influence of the change of the four assembly misalignment on the transmission error curve is as follows:

axis shaft error > axial error of pinion > axial error of gear > offset error of axis

When the assembly misalignment exceed the limit deviation, the edge contact will cause the stress concentration of spiral bevel gears to be serious, and the meshing performance will decline sharply. In this paper, the relationship between the assembly misalignment and the meshing performance of the spiral bevel gears is discussed on the premise that the spiral bevel gears does not have an edge contact. 
In the process of assembly of spiral bevel gears, using the above conclusions can quickly adjust the assembly misalignment, the spiral bevel gears in the best installation location (position of contact area is reasonable and the transmission error curve symmetry), improve the meshing performance of gears. The optimization of the installation position is of great significance for reducing the vibration and noise and improving the transmission stability of spiral bevel gears.

\section{Conclusion}

Through the establishment of meshing model of a pair of spiral bevel gears with assembly misalignment, taking a pair of spiral bevel gears as an example, making tooth contact analysis (TCA) with assembly misalignment, draw contact trace and transmission error curve, the assembly misalignment performance influence of gear meshing simulation results. The quantitative change of contact area and transmission error curve caused by adjusting assembly misalignment.

The influence law summarized in this paper is of great significance for further study of the optimal installation location and its rapid adjustment method. At the same time, the method of determining the variation range of assembly misalignment and the influence of assembly misalignment on the meshing performance of gears can be further extended to other spiral bevel gears.

\section{Acknowledgements}

This paper is supported by the National Natural Science Foundation of China, 51575161 and the National Natural Science Foundation of China, 51475141.

\section{References}

[1] Z.H. Wang, Li. Gang, Kubo. Aizoh. Tooth Contact Analysis of Spiral Bevel Gears Based on Digital Real Tooth Surfaces, J. Journal of Mechanical Engineering, Vol. 50(2014) No.2, p. 1.

[2] Guanglei Liu, Fan, Hongwei1, Gu. Jihong. An Optimization Approach of Transmission Performance of Spiral Bevel Gears, J. Acta Aeronautica ET Astronautica Sinica, Vol. 31(2010) No.8, p. 1680.

[3] J.F. Du, Z.D. Fang, H.B Gao. Contact Analysis of Real Tooth Surface of Hypoid Gear with Cycloid Tooth, J. Journal of South China University of Technology (Natural Science), Vol. 43(2015) No.3, p. 35.

[4] Z.D. Fang, L.L. Guo, J.Z. Su. Optimal Design of Tolerance for Spiral Bevel Gears by Controlling Contact Path, J. Transactions of the Chinese Society of Agricultural Machinery, Vol. 42(2011) No.7, p. 223.

[5] Y.B. Zhuo, X.Y. Xiang, X.J. Zhou. A Method for the Global Optimization of the Tooth Contact Pattern and Transmission Error of Spiral Bevel and Hypoid Gears, J. Journal of Zhejiang University: Science A, Vol. 18(2017) No.5, p. 337.

[6] Aijun Xu. Meshing State Analysis and Experimental Research of Spiral Bevel Gear Based on Driving Error (Ph.D., Xi'an: Northwestern Polytechnical University, China 2012), p.28(In Chinese).

[7] F.L. Litvin. Gear Geometry and Applied Theory (Shanghai Science and Technology Press, China 2008). p.37.

[8] X.M. Cao, N. Sun, X.Z. Deng. Design and Experimental Verification of Low Assembly Misalignment Sensitivity of Spur Bevel Gear, J. Journal of Aerospace Power, Vol. 31(2016) No.1, p. 227(In Chinese).

[9] Y.B. Zuo. Analysis Method and Experimental Research on Quasi Static Contact Characteristics of Spiral Bevel Gear (Ph.D., Zhejiang University, China 2016), p.53 (In Chinese). 